\title{
Components of competitor priming in task switching
}

\author{
Morgan L. Teskey $^{1} \cdot$ Michael E. J. Masson $^{1}$
}

Published online: 17 July 2017

(C) Psychonomic Society, Inc. 2017

\begin{abstract}
Executing an action in response to a stimulus is thought to result in the creation of an event code that integrates stimulus and action features (Allport, 1987; Hommel in Visual Cognition 5: 183-216, 1998). When switching between tasks, competitor priming occurs if a distractor stimulus cues the retrieval of a previously established event code in which that distractor is bound to a competing task, creating a source of interference with the current task whereby the observer is encouraged to apply the competing task to the distractor. We propose a second aspect of competitor priming: the misapplication of the retrieved competing task to the target stimulus. We report two task-switching experiments in which tasks applied to picture-word compound stimuli were manipulated to create conditions in which this second aspect of competitor priming could be revealed and distinguished from other sources of task- and stimulus-based priming. A substantial increase in competitor priming was observed when subjects switched between tasks that required very different processing operations and the competing task was highly relevant to the target stimulus. These results are consistent with our claim that competitor priming can result from applying the competing task either to the distractor that cued it or to the target stimulus.
\end{abstract}

Keywords Competitor priming $\cdot$ Event codes $\cdot$ Task switching

A number of different response options exist when a stimulus is encountered. How a particular response is ultimately

Michael E. J. Masson

mmasson@uvic.ca

1 Department of Psychology, University of Victoria, P.O. Box 1700 STN CSC, Victoria, BC V8W 2Y2, Canada selected will be driven not only by the response options the stimulus affords and the current task demands, but also by previous experiences. Response-selection processes can be studied using the task-switching paradigm, in which subjects alternate between performing two tasks (e.g., Allport, Styles, \& Hsieh, 1994; Rogers \& Monsell, 1995). In situations where subjects switch between tasks, the history of the tasks or stimuli can be manipulated, such that the impact of previous experience on present task performance may be assessed (e.g., Waszak, Hommel, \& Allport, 2005).

Task switching involves shifting mental representations and processes to adapt to a changing set of task instructions - the task set (Rogers \& Monsell, 1995). Evidence for task-set priming has been obtained through the measurement of costs associated with switching tasks. These so-called switch costs present as longer response times on switch trials compared to trials where the task repeats (Rogers \& Monsell, 1995). By comparing switch costs across tasks, the manner in which previous task sets compete with present task sets can be observed. Our interest here is not in switch costs per se, but instead we set out to examine the influence that specific experiences with stimulus items can have on task performance when task sets are rendered unstable by switching between tasks.

For example, an experiment by Allport et al. (1994) used two sets of stimuli: colored words and clusters of digits. In an initial phase, subjects switched between the tasks of reading a word or naming a digit and no switch costs were present. In the next phase of the experiment, these same subjects were required to switch between naming the color of a printed word and counting the number of digits present in a cluster, ignoring the word and digit label that they reported in the initial phase. Under these circumstances, switch costs when alternating between color naming and digit counting were now present. These results suggest that the history of applying a task to a 
particular stimuli class may later prime that associated (now irrelevant) task, leading to interference with one's ability to respond correctly to a stimulus of that class under the current task set.

\section{Item-specific priming}

Waszak, Hommel, and Allport (2003) aimed to extend the view of priming developed by Allport et al. (1994) to include the potential for experience with a specific stimulus, rather than an entire stimulus class, to interfere with task performance. The stimuli they used were picture-word compounds created by superimposing a word over a line drawing of a semantically related object. One of the alternating tasks required naming the depicted object, and the other required reading aloud the word. Switch costs were greater when shifting to the task of reading a word if the compound stimulus of it was a part that had been seen previously in the context of the picture-naming task. Waszak et al. proposed that the task set that is active during the presentation of a stimulus becomes associated with that stimulus. These stimulus-task associations or episodes are particularly influential during switch trials, when the intended task set is weak, relative to a repeat trial where the relevant task set is well established (see also Koch \& Allport, 2006; Waszak et al., 2005). This finding suggests that previous experience with a stimulus can invoke a task set that is not relevant under the current instruction.

There are two distinct forms of priming that may have driven the priming effects found by Waszak et al. (2003). First, having previously responded to one element of the current picture-word compound interferes with making a response if the other element of the compound is currently the target stimulus. For example, if shown a picture of a shoe with the word HORSE superimposed and given the task of reading the word, performance is slower if the picture of a shoe appeared earlier on a picture-naming trial. This effect is known as competitor priming. The second factor is negative priming, in which a response is to be made to a stimulus that in a previous trial was ignored (Neill, 1977; Tipper, 1985).

Waszak et al. (2005) explored each of these of item-specific priming effects when switching between word-reading and picture-naming tasks. Picture-word compounds were presented in such a way that negative and competitor priming could be evaluated individually and in conjunction. Taking the word-reading task as the task of interest, a condition in which both elements of the compound had been presented only in word-reading trials acted as a baseline, unprimed condition. Negative priming was created by forming compounds in which the picture was seen only in word-reading trials, but the word was seen in both picture-naming and word-reading trials. On picture-naming trials the word-reading response would presumably be suppressed so that when a reading response was subsequently required on a word-reading trial, negative priming would be expressed. Competitor priming involved compounds where the picture was seen in the context of both tasks, paired with a word seen only in the word task. Reading a word in the context of a picture that previously had been named creates the opportunity for the picture to retrieve a stimulus-task episode involving picture naming, which would interfere with the current task goal of reading the word. Finally, an item comprised of picture and word elements that were both seen in the context of both picture-naming and word-reading tasks could generate both negative and competitor priming.

Waszak et al. (2005) found evidence for competitor priming, but not for negative priming, unless a small number of unique stimuli were used, which is consistent with other work on negative priming (e.g., Malley \& Strayer, 1995). Competitor and negative priming stand as item-specific contributions to total priming effects that occur in addition to other aspects of switch cost that are captured by the unprimed condition in the Waszak et al. experiments.

\section{Stimulus-task episodes}

Waszak and colleagues (e.g., Waszak \& Hommel, 2007; Waszak et al., 2003, 2004) proposed that competitor and negative priming effects are produced by stimulus-task episodes formed on previous trials. For example, executing a goal-directed action such as naming a picture that is part of a picture-word compound is assumed to create an integrated episode that binds information about the stimulus, the action performed on it, and the taskrelated goals of that action (Allport, 1987; Hommel, 1998, 2004, 2009; Koch \& Allport, 2006). When that picture later appears as part of a compound on a wordreading trial, it triggers retrieval of the earlier picturenaming episode, which then produces competitor priming. A similar process would account for negative priming, whereby the appearance of a word on a picturenaming trial would bind that word stimulus with the task goal of not making a response to it. Evidence that episodic binding of stimulus and task goes beyond basic stimulus-response pairing was provided by Koch and Allport (2006) and Waszak and Hommel (2007), who showed that when subjects made binary key-press responses to classify stimuli in two different tasks, priming effects were substantial even when the target stimulus called for the same key-press response for either task (see also Waszak et al., 2003).

This proposal regarding stimulus-task episodes implies that in the case of competitor priming, the competing task set is associated with the currently irrelevant stimulus. The interference that occurs is a product of a tendency to make a response, 
based on the competing task set, to the distractor element of the current stimulus compound. For example, Waszak et al. (2005) described competitor priming when a distractor picture and word target are presented as being due to reactivation of "the (now inappropriate) picture-naming process in response to the current (picture) distractor" (p. 597). If we assume that a distractor stimulus is associated not only with its task-relevant response, but also with the task set that determined that response, then there may be an additional mechanism that contributes to competitor priming. Specifically, there may be a tendency for the competing task, called forward by the distractor stimulus element, to be misapplied to the target stimulus. For example, reading the word component of a picture-word compound might be slowed in part because previous experience applying a rather different task to the picture component inclines the subject to apply that task (instead of the reading task) to the word target on the current trial. This process would amount to a decoupling of the components of the stimulus-task episode recruited by the distractor stimulus, and would imply a limit on the cohesion of the episodic representation. Consistent with this possibility, Hommel (2004) characterized the integration of stimulus components and tasks into episodes, or event files, as a collection of "binary bindings, a loose network of clusters rather than one master file" (p. 495).

In previous work using picture-word compounds, both picture and word tasks involved producing the phonological code associated with the target item word reading and picture naming (e.g., Waszak et al., 2003, 2004, 2005). In these situations, the misapplication of the competing task to the target stimulus would elicit the same response as the application of the current task to the target. This arrangement affords no opportunity to distinguish between the potential application of the competitor task to the distractor stimulus versus the target stimulus. Therefore, to test our proposal that a second source of competitor priming exists, we required subjects to apply quite different tasks to picture versus word targets. We used picture-word stimulus compounds as in Waszak et al. (2005), but we also introduced a task that required subjects to make a manual response to classify a stimulus as representing a living or nonliving object. By requiring subjects to switch between two tasks requiring very different responses (e.g., classifying pictures and reading words), we created a situation in which we could distinguish our proposed additional source of competitor priming from the established source.

We anticipated that if subjects mistakenly attempt to apply a completely incompatible task to the target stimulus, then greater interference would be expected, resulting in exaggerated competitor priming.

\section{Experiment 1}

Two versions of Experiment 1 were conducted. One was a replication of the Waszak et al. (2005) study in which subjects switched between naming pictures and reading words (Exp. 1A). Unlike Waszak et al. (2005), however, we included an unprimed condition for picture-naming trials so that we could assess competitor and negative priming not only for the word task, but also for the picture task. In the other version of the experiment (Exp. 1B), instead of naming pictures, subjects performed a semantic classification task in which they pressed response buttons to indicate whether pictures represented living or nonliving objects. The task applied to word targets was again word reading. By making the nature of the tasks associated with pictures and words very different, we created the opportunity for competitor priming to have an enhanced influence by inducing subjects to apply the wrong (and strongly conflicting) task set to the target stimulus. This result should occur if previously encoded stimulus-task episodes generate competitor priming not only by encouraging re-enactment of the original episode, but also by prompting the application of the competing task to the current target stimulus.

\section{Method}

\section{Subjects}

Ninety-six students at the University of Victoria participated for extra credit in an undergraduate psychology course. Half participated in Experiment 1A and half in Experiment 1B. Testing in these two sub-experiments was interleaved, but group assignment was not completely random.

\section{Materials and design}

Picture-word compounds were created by overlaying words on line drawings. Elements for these compounds consisted of 24 semantically unrelated stimuli. Half of these stimuli represented non-living objects (e.g., book, cake, shoe) and the other half represented living things (e.g., bird, camel, frog). Twelve stimuli were randomly assigned to be pictures and 12 were words, with six living and six non-living items of each type. No pictured object was also presented as a word, and vice versa. Pictures of objects were line drawings taken from the Snodgrass and Vanderwart (1980) standardized set of pictures. These elements were combined and recombined to form picture-word compounds in such a way that all four priming conditions could be established for the word and for the picture tasks: unprimed (UP), negative priming (NP), competitor priming $(\mathrm{CP})$, and negative-and-competitor priming (NCP). There was no constraint that a particular word always be paired with a particular picture. Three different assignments 
of items to conditions were used and equal numbers of subjects were tested with each one.

Priming conditions were established by presenting four of the pictures only when the picture was the target stimulus, four pictures only when the word was the target, and four pictures on both types of trial. The word stimuli were also arranged in this manner. Therefore, for the word task, pairing a "word-task only" picture with a "word-task only" word created an unprimed stimulus (UP condition). When a "word-task only" picture was combined with a word that occurred in both the picture and the word task, this item was in the NP condition. When the picture was seen in both the picture and the word task and the word was in the "word-task only" category, the resulting compound was in the $\mathrm{CP}$ condition. Finally, the NCP condition consisted of trials where both the picture and the word were seen in both tasks. For the picture task, the four priming conditions were constructed in the same way, with picture and word tasks reversing roles (e.g., the UP condition consisted of compounds in which the pictures and words appeared only in the picture task).

Task sequences were established as two-trial runs, meaning a particular task was performed for two consecutive trials, then a switch was made to the other task for two trials before switching again. Each task switch was signified by a letter that cued the task set for the following two trials. The use of a task cue on switch, but not repeat, trials introduces a confound with respect to comparing performance on switch and repeat trials because all switch task trials are preceded by a cue, creating a longer duration between the previous trial and a switch trial than between the previous trial and a repeat trial. We adopted this methodology from Waszak et al. (2005) so that we could replicate their procedure and assess competitor and negative priming under conditions very similar to theirs. We recognize that this design limits our ability to interpret switch cost results, but it does not impact the interpretation of our primary measure of interest, which is competitor and negative priming effects occurring within switch trials or within repeat trials.

\section{Procedure}

Subjects were tested individually in a quiet room using a Macintosh desktop computer under the supervision of an experimenter. Vocal responses were scored as correct or incorrect at the end of the trial by the experimenter. The experiment was controlled by a program implemented using the SuperLab application for the Apple operating system, which required $50 \mathrm{~ms}$ of continuous vocal input for a vocal response to be recorded. Any non-response vocalizations or hesitations were marked as spoiled trials at the discretion of the experimenter. Clear vocal responses that did not correspond to the target were classified as errors. In Experiment 1A, subjects made a vocal response to the target stimulus, either naming the picture or reading the word. In Experiment 1B, subjects read the word or classified the picture as living or non-living with a left- or right-handed button press on a response box. Button assignment was counterbalanced across subjects.

Trials were presented in pairs, with the first trial of a pair preceded by a cue, the letter $P$ or $W$, indicating the task (picture naming or word reading in Exp. 1A, picture classification or word reading in Exp. 1B) to be performed on the upcoming two trials. The cued task was changed after every pair of trials, so the first trial in a pair always constituted a task switch and the second trial in the pair repeated the task. Figure 1 illustrates the events for an example pair of trials. Each sequence of two trials began with a fixation cross on the computer monitor that was in view for $500 \mathrm{~ms}$, followed by task cue consisting of an uppercase $P$ or $W$. The cue was visible for $1,000 \mathrm{~ms}$ then was replaced by a blank display for $500 \mathrm{~ms}$, followed by the picture-word compound for the first trial. After a response was made, the display was blank for $500 \mathrm{~ms}$, then the stimulus for the second trial was presented until a response was made. The next pair of trials was presented $500 \mathrm{~ms}$ after that response.

A cycle of four trials consisted of two picture-task trials followed by two word-task trials. Each block of trials consisted of four of these four-trial cycles, and included two trials for each priming condition (NCP, CP, NP, and UP) in each of the two tasks (picture, word). Stimulus compounds constituting the four priming conditions for each task were presented in a random order within each block. Over the course of the full set of trials (21 blocks), each priming condition was tested equally often on switch and repeat trials. The first two blocks (32 trials) were treated as practice trials and allowed subjects to have an initial relevant experience with the individual stimuli (i.e., picture task, word task, or both). These trials were not included in the analyses. The remaining 19 blocks (304 trials) were the critical trials. Over the course of the critical trials, the lag between repetitions of a particular word stimulus ranged from 1-85 trials with a median lag of 9 . For picture stimuli, the range of lags between repetitions of an item was 1-64 with a median of 9 .

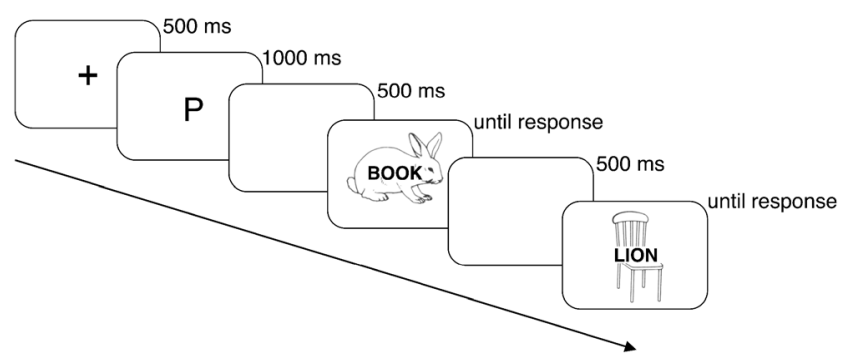

Fig. 1 An example of a two-trial sequence for the picture task in Experiment 1 
Table 1 Mean percent error $(\% \mathrm{E})$ and spoiled $(\% \mathrm{~S})$ trials in each condition of Experiments 1 and 2

\begin{tabular}{|c|c|c|c|c|c|c|c|c|c|c|c|c|c|c|c|c|}
\hline \multirow[b]{3}{*}{ Exp. } & \multicolumn{8}{|c|}{ Word task } & \multicolumn{8}{|c|}{ Picture task } \\
\hline & \multicolumn{4}{|l|}{ Switch } & \multicolumn{4}{|c|}{ Repeat } & \multicolumn{4}{|l|}{ Switch } & \multicolumn{4}{|c|}{ Repeat } \\
\hline & $\mathrm{NCP}$ & $\mathrm{CP}$ & $\mathrm{NP}$ & UP & $\mathrm{NCP}$ & $\mathrm{CP}$ & $\mathrm{NP}$ & UP & $\mathrm{NCP}$ & $\mathrm{CP}$ & $\mathrm{NP}$ & UP & $\mathrm{NCP}$ & $\mathrm{CP}$ & NP & UP \\
\hline \multicolumn{17}{|c|}{ 1A: word naming, picture naming } \\
\hline$\% \mathrm{E}$ & 1.6 & 0.5 & 0.4 & 0.3 & 0.4 & 1.3 & 0.1 & 0.0 & 1.2 & 0.7 & 1.3 & 1.1 & 1.1 & 1.6 & 1.2 & 0.5 \\
\hline$\% \mathrm{~S}$ & 3.1 & 1.3 & 1.5 & 1.9 & 3.1 & 2.4 & 3.0 & 3.3 & 3.5 & 3.8 & 3.2 & 3.2 & 4.0 & 5.0 & 3.9 & 4.4 \\
\hline \multicolumn{17}{|c|}{ 1B: word naming, picture classification } \\
\hline$\% \mathrm{E}$ & 0.0 & 0.0 & 0.0 & 0.0 & 0.0 & 0.0 & 0.0 & 0.0 & 3.4 & 1.9 & 3.5 & 1.2 & 1.3 & 1.8 & 2.3 & 1.1 \\
\hline$\% \mathrm{~S}$ & 3.2 & 2.6 & 3.7 & 2.6 & 7.1 & 7.4 & 7.5 & 6.8 & - & - & - & - & - & - & - & - \\
\hline \multicolumn{17}{|c|}{ 2A: word classification, picture naming } \\
\hline$\% \mathrm{E}$ & 3.7 & 3.4 & 3.1 & 3.0 & 1.9 & 2.1 & 2.2 & 2.3 & 0.8 & 0.3 & 0.9 & 0.3 & 0.2 & 0.2 & 0.5 & 0.1 \\
\hline$\% \mathrm{~S}$ & - & - & - & - & - & - & - & - & 5.1 & 4.4 & 4.6 & 6.5 & 8.9 & 6.3 & 7.9 & 10.2 \\
\hline \multicolumn{17}{|c|}{ 2B: word classification, picture classification } \\
\hline$\% \mathrm{E}$ & 5.5 & 4.5 & 4.5 & 3.2 & 5.6 & 6.6 & 4.3 & 3.8 & 3.2 & 3.5 & 3.4 & 3.3 & 2.7 & 1.7 & 3.7 & 3.2 \\
\hline
\end{tabular}

Note. Spoiled trials were not possible when the classification task was used because it required manual responses (picture task in Exp. 1B, word task in Exp. 2A, both tasks in Exp. 2B)

\section{Results}

Mean percent error and mean percent spoiled trials in each condition are shown in Table 1. Overall error and spoil rates were low and statistical analyses indicated that the pattern of errors did not indicate the presence of speed-accuracy tradeoffs that would compromise the interpretation of the responsetime data. Full analyses of these dependent measures are presented in the Appendix. The absence of any errors in the wordnaming task of Experiment 1B is not very surprising given that pictures were never named in that experiment and that only a small set of repeatedly presented words was used.

Response times less than $200 \mathrm{~ms}$ or greater than $1,800 \mathrm{~ms}$ in Experiment 1A or 2,000 ms in Experiment 1B were excluded as outliers. The upper boundaries were defined so that no more than $0.5 \%$ of observations were excluded (Ulrich \& Miller, 1994). Trials following a spoil or error response were included in our analyses. Mean correct response time in each condition is shown in Fig. 2. The effects of task switching and priming across the picture and word tasks were assessed by computing the Bayes factor favoring either a model that included the effect of interest or a model that excluded that effect. These Bayes factors were generated using the anovaBF function in the BayesFactor package in R (Morey \& Rouder, 2015; Rouder, Morey, Verhagen, Swagman, \& Wagenmakers, 2017; see Rouder, Morey, Speckman, \& Province, 2012, for the theoretical foundation of the methods used in this package). The default priors in this package for alternative hypotheses in factorial designs were used. We specified 100,000 iterations for convergence and we selected the whichModels = "top" option for the analysis of effects when more than one independent variable was included in the analysis. This option involves comparing a model that includes all of the effects in the design against a model that excludes one specific effect, with each particular effect considered in turn. If the model excluding the effect of interest is preferred over the model that includes it, then we have evidence favoring a null effect. If the model that includes the effect (the full model) is favored, however, then we have evidence in support of that effect. This analysis produces a Bayes factor $(B F)$ for each effect, supporting either its presence or absence. Bayes factors of 3 or greater are generally taken as positive support for an outcome, and a Bayes factor of 20 or more indicates at least strong support (Raftery, 1995). When we report a Bayes factor that indicates the presence of an effect, that effect would also be significant in a standard analysis of variance with a $p$ value of .05 or less. In addition to condition means, the means for the three types of priming effects, competitor, negative, and both combined, are shown in Fig. 3. Each effect is plotted with the 95\% most plausible estimated values for effect size (highest probability interval) based on a Bayesian analysis using the ttest $B F$ function in the BayesFactor package with the default prior distribution and 100,000 iterations.

To establish that our experiment generated typical task switch costs, we first compared the effects of task switching across the picture and word tasks of Experiments 1A and 1B separately. To do this, we averaged across the four priming conditions to obtain an overall mean response time and used that as the dependent measure. A Bayesian analysis with trial type (switch, repeat) and target type (word, picture) as factors was computed for each experiment. For Experiment 1A, there was a robust task-switch cost indicated by longer response time on switch trials $(B F>1,000)$, and this effect was greater 
Experiment $1 \mathrm{~A}$

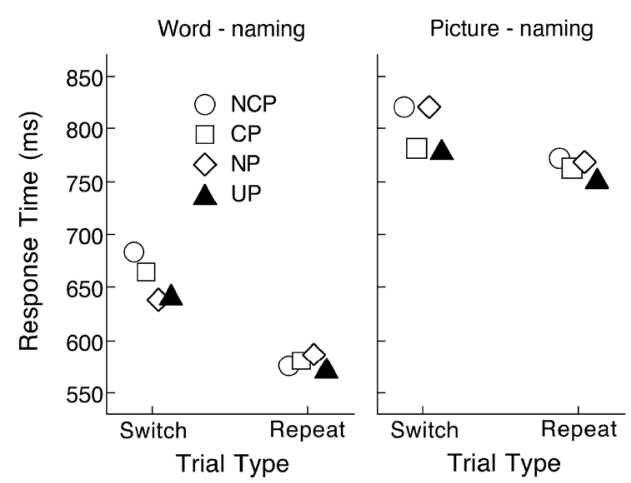

Experiment 2A

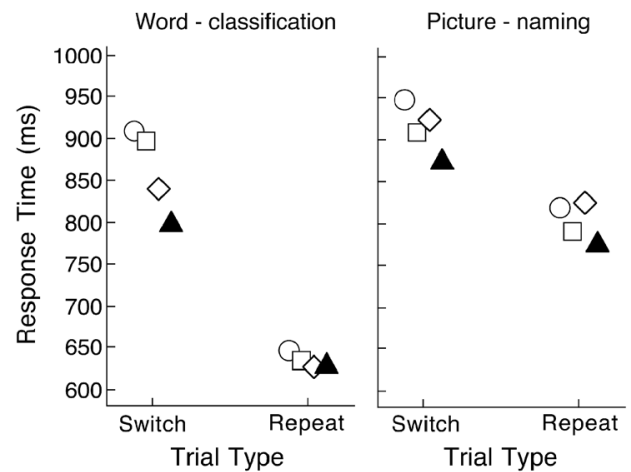

Experiment 1B

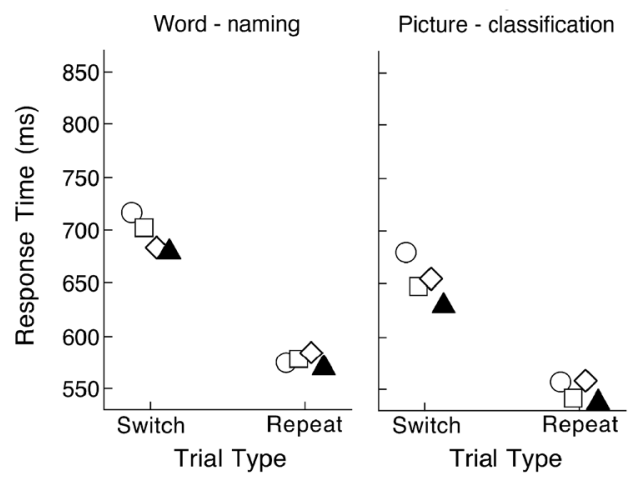

Experiment 2B

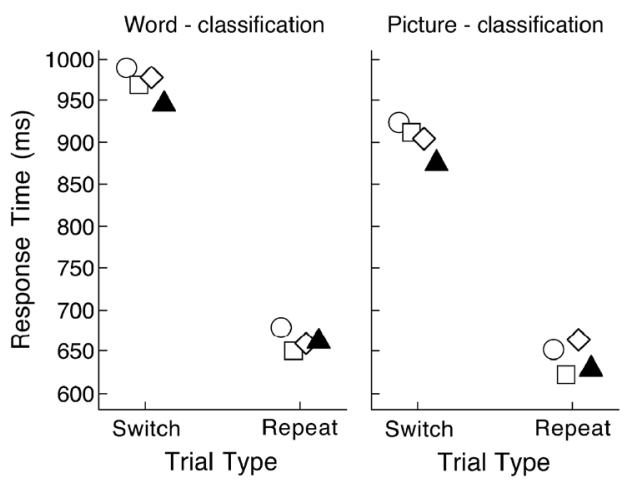

Fig. 2 Mean response times as a function of prime type, trial type, and target type for Experiments 1 and 2. $N C P$ negative and competitor prime, $C P$ competitor prime, $N P$ negative prime, $U P$ unprimed

for word reading (78 $\mathrm{ms})$ than for picture naming $(36 \mathrm{~ms})$ as revealed by an interaction between trial type and target type $(B F=18.2)$. Response times were also much longer for picture-naming than for word-reading $(B F>1,000)$, in keeping with the finding that subjects more readily apply phonological encoding to words than generate the name code for a picture (Smith \& Magee, 1980). These effects replicate the Waszak et al. (2005) findings and are similar to the pattern with the color-word Stroop task-switching results reported by Allport and Wylie (1999, 2000), indicating that the wordreading task is dominant relative to the picture-naming task (i.e., shorter response times, but greater switch cost). In Experiment 1B, where the picture task was changed to semantic classification, once again there was a large task-switch cost $(B F>1,000)$, but switch cost did not reliably vary across the picture and word targets (105 ms and $118 \mathrm{~ms}$, respectively), with the Bayesian analysis favoring the null hypothesis $(B F=$ 3.7). Responses were faster in the picture task than in the word task $(B F=206.7)$.

Next, we asked whether priming effects varied across switch versus repeat trials, with more robust priming on switch trials, as was seen in Waszak et al. (2003, 2005). The priming effects shown in Fig. 3 suggest that this pattern emerged here as well. We tested the influence of task switching on the priming effect using the theoretically maximal priming effect, based on the NCP condition, which included possible influences of both negative and competitor priming. NCP priming was computed as the difference between the NCP and the UP conditions and was used as the dependent variable in a Bayesian analysis with trial type (switch, repeat) and target type (picture, word) as factors. For Experiment $1 \mathrm{~A}$, the NCP priming effect was reliably greater for switch than for repeat trials, averaging across the two tasks $(B F=54.2)$, and was of similar magnitude for both tasks $(B F=$ 4.0 favoring the null hypothesis for the test of the effect of target type). Experiment 1B produced the same outcome $(B F=181.8$ for the effect of task switch on priming magnitude, and $B F=1.7$ for the lack of an effect of target type). These results show that priming effects are stronger when task competition is particularly strong, as on switch trials. Moreover, Hommel (2004) reviewed evidence showing that an object will prime its event file to a degree that is weighted by task relevance. On a switch trial, the previously executed task will have greater relevance than on a repeat trial, at which point the new task set will have been established. Consequently, on the event file account, episodes associated with a distractor stimulus are more likely to be recruited on a switch trial.

Figure 3 also shows that on switch trials in Experiment 1A, competitor priming was somewhat more evident than negative 

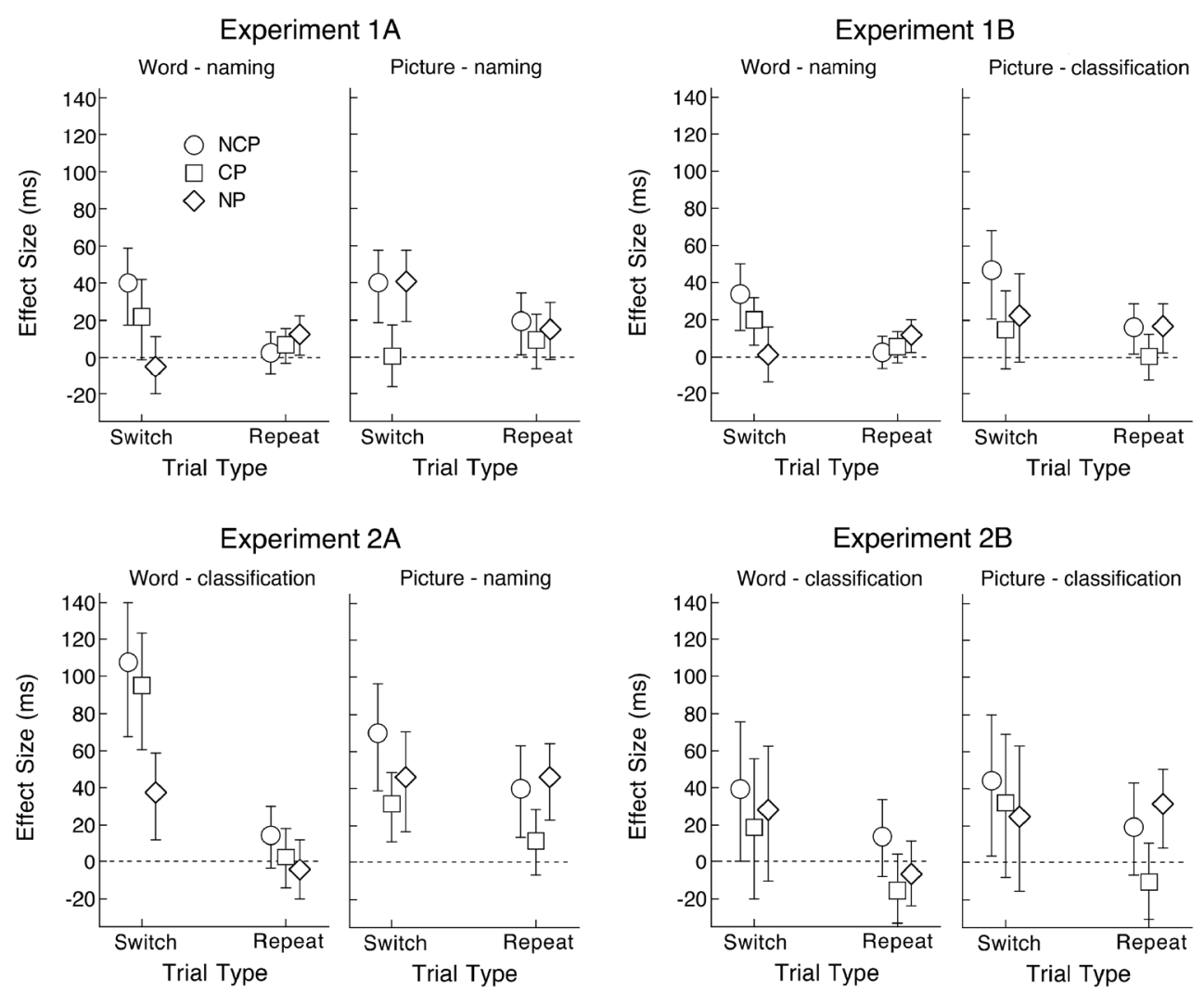

Fig. 3 Mean priming effects as a function of prime type, trial type, and target type for Experiments 1 and 2. Error bars represent $95 \%$ highest probability intervals. $N C P$ negative and competitor prime, $C P$ competitor prime, $N P$ negative prime

priming on the word task, but the reverse was true for picture priming. The small difference between the two types of priming was not reliable for words ( $B F=1.5$ favoring a difference), but it was reliable for pictures $(B F=212.5)$. The former result is consistent with Waszak et al. (2005, Exp. 2), where the same number of unique word and picture stimuli were used (12 of each) as in this experiment (negative priming is known to be sensitive to the size of the stimulus set; Malley \& Strayer, 1995). Waszak et al. also reported a nonsignificant tendency for negative primed pictures to have longer responses times than competitor primed pictures (recall that they did not include a UP condition in their picture task and so could not measure priming relative to an unprimed condition). Our finding of greater negative than competitor priming for pictures is larger than the difference they found, but it is not clear why this difference was exaggerated in our case. In many respects, however, our Experiment 1A replicated the results reported by Waszak et al.

Our primary interest was in the question of whether competitor priming would be enhanced (or possibly reduced) when the tasks applied to picture and word stimuli required very different processing operations. The goal was to obtain evidence for the possibility that a competitor-primed task set might be directed toward the target stimulus, creating a source of interference additional to that generated by the episodically bound pairing of competitor task and distractor stimulus. This hypothesis was tested by comparing the size of competitor priming effects across Experiments 1A and 1B. In the former, the picture and word tasks were very similar (naming the stimulus), whereas in the latter, the task requirements diverged substantially (word reading vs. picture classification). A Bayesian analysis of response times on switch trials (where priming effects are maximal) with experiment $(1 \mathrm{~A}, 1 \mathrm{~B})$ and prime condition (CP, UP) as factors was computed separately for the word and the picture targets. For word targets, there was clear evidence for competitor priming ( $B F=14.9)$, but no indication that the effect varied across the two experiments ( $B F=4.8$ favoring the null hypothesis). For picture targets, there was no reliable competitor priming, and no indication of variation in this priming across experiments $(B F>2.8$ favoring the null hypothesis in both cases).

\section{Discussion}

Changing the nature of the picture task in Experiment 1B to be very different from the word task led to an increase in the switch cost, particularly for the picture task, relative to Experiment 1A. The classification task was performed much more efficiently than the naming task for picture targets, making for a similar level of difficulty for the word and picture tasks in Experiment 1B. In a situation without task dominance, a symmetrical switch cost is not surprising (e.g., 
Allport et al., 1994). The larger switch cost in Experiment 1B may be a result of subjects having to switch not only between stimulus class (word/picture), but also between response rules (name/classify). It is well known that switching response rules while holding the stimulus class constant across trials will induce a large switch cost (e.g., Jersild, 1927; Koch, Prinz, \& Allport, 2005).

By using a different task for word and picture targets in Experiment 1B, we created an opportunity to examine whether misapplication of the competitor task to the target stimulus impacts the magnitude of competitor priming. We hypothesized that a task set activated through priming of previous processing episodes involving application of the picture task to the current picture distractor stimulus could lead to an erroneous application of that task set to the current target stimulus (word). Using a competing task set that requires very different cognitive operations than the task set to be applied to the target stimulus might lead to exaggerated interference if the subject is inclined to apply the inappropriate task to the target stimulus. Alternatively, a decrease in competitor priming could be seen if switching between two distinct task sets allows subjects to more reliably maintain a separation between the tasks sets and thereby experience a reduced tendency to misapply the inappropriate task to the target stimulus. The results indicated that no increase or decrease in competitor priming occurred when the picture task was changed from naming to semantic classification.

There are two possible explanations for this outcome. First, episodic bindings of components of earlier processing episodes (e.g., naming a picture) may be so strongly integrated that the included task set is not likely to be mistakenly applied to a different stimulus when that episode is recruited by the current distractor stimulus. This constraint would mean that competitor priming is driven by one's propensity to re-enact a previous episode (stimulus-task pair), but not by any tendency to apply the primed task set to the target stimulus. Alternatively, the primed task set may have the potential to be misapplied to the target stimulus, but only in situations where that task is naturally afforded by the target stimulus. For word targets, classifying items as living or non-living is not an activity that is commonly experienced and perhaps this is why the classification task did not appear to be misapplied to word targets. In contrast, an operation that is naturally and, in some sense, automatically applied to words is reading. Although subjects do not always automatically read words that appear as background stimuli, distributing visual attention across the word is likely to invite its reading as a default response (Besner \& Stolz, 1999; Stolz \& Besner, 1996).

With this possible constraint in mind, we considered that in Experiment 1 the semantic classification task might not have been a strong competitor for the reading task that was to be applied to word targets. Word stimuli naturally afford reading (MacLeod, 1991; Stroop, 1935) and the propensity to read a word target might well create interference for an intended, less fluent task that is to be applied to a word target. In a second experiment, therefore, the nominal word task was changed to semantic classification and the picture task was either naming (Exp. 2A) or classification (Exp. 2B). In this case, it was expected that when pictures were named, competitor priming on word classification trials could be supplemented by the tendency to apply the competitor task (naming) to the target stimulus (word), in opposition to the required classification task. This additional source of competition should yield a greater competitor priming effect than that obtained when the picture task requires semantic classification instead of naming.

\section{Experiment 2}

Experiment 2 was a replication of Experiment 1, except that the word task in both versions of this experiment was semantic classification.

\section{Method}

\section{Subjects}

Ninety-six new subjects from the same source as in Experiment 1 participated in this study. An equal number of subjects participated in Experiments 2A and 2B. As in Experiment 1, testing in these two sub-experiments was interleaved although subjects were not fully subject to random assignment to sub-experiment.

\section{Procedure}

The stimuli, design, and procedure were the same as in Experiment 1 with the following exceptions. In Experiment 2A subjects made either a vocal response to name a picture or a button press to classify a word as living or non-living. In Experiment 2B subjects made button-press responses to classify a picture or word target as living or non-living.

\section{Results}

Mean error percent and spoil percent for each condition are shown in Table 1. As in Experiment 1, error and spoil rates were low and the pattern of results did not contradict any of the outcomes of the analyses of response time that we report. Response times shorter than $200 \mathrm{~ms}$ or greater than $2,400 \mathrm{~ms}$ in Experiment 2A or 3,200 ms in Experiment 2B were considered outliers and excluded from the analyses. The upper limits were set so that no more than $0.5 \%$ of correct responses were excluded. We included in our analyses correct responses from trials that followed a trial classified as an error or spoil. 
Mean correct response time for each condition is shown in Fig. 2. Response time data were first examined in separate Bayesian analyses for Experiments $2 \mathrm{~A}$ and $2 \mathrm{~B}$ that included trial type and target type as factors. In the initial analyses, we collapsed across prime condition. In Experiment 2A, there was a substantial switch cost, but this cost was larger for the word task $(227 \mathrm{~ms})$ than for the picture task $(111 \mathrm{~ms})$ and responses were generally faster on the word task as well ( $B F$ $>1,000$ in all cases). In Experiment 2B, where the classification task was applied to both target types, there was a large switch cost $(B F>1,000)$, but now responses were faster to pictures than to words $(B F=17.0)$ and there was no evidence of a difference in switch cost for the two types of target (308 ms for words, $262 \mathrm{~ms}$ for pictures; $B F=1.3$ favoring the null hypothesis). The generally larger switch costs in Experiment 2 relative to Experiment 1 might reflect differences in input-output compatibility. ${ }^{1}$ Stephan and Koch (2010, 2011) have shown that switch costs are smaller when the modality of stimulus input and response output for a task are compatible (e.g., auditory input paired with vocal output) rather than incompatible (e.g., auditory input, manual output). Arguably, the word-naming/picture-classification arrangement in Experiment $1 \mathrm{~B}$ is a more compatible pairing of inputs and outputs than the opposite pairing that was used in Experiment 2A.

Switch costs were particularly high in Experiment 2B where switching occurred between classification tasks. This arrangement creates the possibility of enhanced switch cost due to a possible conflict between the classification responses implied by the two competing stimuli. For example, a compound involving the word $B O O K$ and a picture of a chair would have compatible nonliving classification responses for both the target and the distractor, and could theoretically lead to a faster response than a stimulus consisting of the word $R A B B I T$ and a picture of a chair, where the classification responses would be incompatible (see Koch \& Allport, 2006; Waszak, 2010; Waszak \& Hommel, 2007). To determine whether this was the case, we compared responsecompatible to response-incompatible trials in an analysis that included trial type, target type, and prime time as additional factors. Results showed that response times were shorter in the response-compatible than in the response-incompatible case (777 ms vs. $810 \mathrm{~ms} ; B F=20.6)$. Although switch cost was somewhat larger on response-compatible trials (297 ms vs. $268 \mathrm{~ms}$ ), the interaction between response compatibility and trial type was not reliable ( $B F=2.6$ in favor of the null model). Response compatibility also did not interact with target type $(B F=5.2$ in favor of the null model). The potential for response conflict could have led subjects to apply a stronger degree of top-down control to "shield" the current task set from distraction, leading to a larger cost when the task set

${ }^{1}$ We thank an anonymous reviewer for suggesting this explanation. must be switched (Dreisbach \& Haider, 2009; Dreisbach \& Wenke, 2011). An alternative possibility is that the classification task, particularly with its response-mapping rules, is an arbitrary one for both types of stimuli and this situation might be an example of incompatible input-output assignments for both tasks, which is known to elevate switch costs (Stephan \& Koch, 2010).

Our primary question in Experiment 2 was whether competitor priming would be enhanced when the word classification task was paired with picture naming. In that case, we anticipated the possibility that the naming task, in the role of competitor on word-classification trials, might be misapplied to the word target, thereby adding a further source of interference with correct task execution. Mean priming effects are shown in Fig. 3. As in Experiment 1, there was evidence in Experiment 2A that priming effects were stronger on switch trials. When both negative and competitor priming acted together (NCP condition), the priming effect (relative to the UP condition) was greater on switch trials than on repeat trials (89 ms vs. $27 \mathrm{~ms} ; B F>1,000$ ). This effect was not reliable in Experiment $2 \mathrm{~B}$ ( $B F=1.8$ favoring the null hypothesis).

On the critical question of whether competitor priming would be increased when the competitor task was readily afforded by the target stimulus, the priming effects for word targets shown in Fig. 3 strongly suggest confirmation of this proposition. A Bayesian analysis of response times to word targets on switch trials, with prime type (CP, UP) and experiment $(2 \mathrm{~A}, 2 \mathrm{~B})$ as factors provided clear evidence that competitor priming of word targets was larger in Experiment $2 \mathrm{~A}$ than in $2 \mathrm{~B}(95 \mathrm{~ms}$ vs. $19 \mathrm{~ms} ; B F=10.6)$. Competitor priming of word targets was also larger in Experiment $2 \mathrm{~A}$ than in either version of Experiment 1 ( $B F \mathrm{~s}>100)$, although we do not draw strong conclusions from this comparison because different word tasks were used in Experiments 1 and 2. It should be emphasized that the increased competitor priming in Experiment 2A was not a general one that occurred also for negative priming. An analysis of negative priming in Experiment $2 \mathrm{~A}$ versus $2 \mathrm{~B}$ found clear evidence for negative priming $(B F=8.0)$, but showed that the effect was the same regardless of the nature of the picture task $(B F=4.3$ favoring the null model). Further, the elevated competitor priming in Experiment 2A is not likely due to the increased switch cost in that experiment compared to Experiment 1, given that the largest switch cost of all, in Experiment 2B, yielded a level of competitor priming comparable to that found in Experiment 1.

We considered the possibility that the effect of competitor priming when responding to picture targets could be augmented by misapplication of the word task to the picture target. This hypothesis was tested by comparing competitor priming in the picture-naming task across Experiments $1 \mathrm{~A}$ and $2 \mathrm{~A}$, and in the classification of pictures across Experiments $1 \mathrm{~B}$ and $2 \mathrm{~B}$. We caution that the validity of these comparisons is 
constrained by the fact that overall response times and switch costs were greater in Experiment 2 than in Experiment 1. In both cases, the nature of the word task varied across experiments and the question was whether the size of competitor priming on switch trials when responding to picture targets would be affected by the nature of the word task. It might be expected that when the word task requires substantially different processing operations relative to the picture task, competitor priming would increase. This would be particularly likely if the word task were one that is frequently applied when interacting with pictures or objects. Neither picture naming nor semantic classification are particularly common operations, so there was no strong expectation that enhanced competitor priming would be observed.

In the comparison between Experiments $1 \mathrm{~A}$ and $2 \mathrm{~A}$, the picture task was naming and there was a tendency for competitor priming to be larger when the word task was classification (relative to word reading), but this trend was not clearly supported by a Bayesian analysis $(B F=2.6)$. In Experiments $1 \mathrm{~B}$ and $2 \mathrm{~B}$, the semantic classification task was applied to picture targets and a Bayesian analysis showed support for equal amounts of competitor priming regardless of the nature of the word task ( $B F=3.6$ favoring the null hypothesis). With pictures as targets, it appears that we did not have a competing task that subjects readily misapplied to those items.

\section{Discussion}

Changing the relationship between the picture and the word tasks used in 2 created an opportunity to examine a component of competitor priming driven by the misapplication of the competing task to a target stimulus that naturally afforded that task. Elevated competitor priming in Experiment 2A compared to $2 \mathrm{~B}$ did not extend to negative priming. Neither was any reliable variation in competitor priming across task manipulations seen when pictures were the target, nor in Experiment 1 when the task competing with naming word targets was either a compatible task (Exp. 1A) or a task not naturally afforded by word processing (Exp. 1B). The specificity of the modulation of competitor priming in Experiment 2 provides convincing evidence that subjects may be induced to apply a competitor task to the current target stimulus, even when that competing task set is part of an episode in which it is bound with the current distractor stimulus.

One might argue that the elevated competitor priming effect seen in Experiment 2A was a result of longer response times or larger switch costs than those seen in Experiment 1. Contrary to this notion, response times and switch costs in Experiment $2 \mathrm{~B}$ were at least as large as in Experiment 2A, yet no amplification of competitor priming was seen in Experiment 2B. As a result, we conclude that the increase to competitor priming seen in Experiment 2A, relative to all three of the other experiments reported here, was a consequence of a tendency to apply the competitor task to the target stimulus.

An additional possibility is that when the tasks associated with the distractor and target stimuli are the same (as in Exp. $1 \mathrm{~A}$ and $2 \mathrm{~B}$ ), any tendency to apply the distractor task to the target stimulus might actually improve performance. If this factor were in play, it might have contributed to the difference in competitor priming seen across Experiments $2 \mathrm{~A}$ and $2 \mathrm{~B}$. That is, application of the distractor task to the target stimulus would potentially increase competitor priming in Experiment 2A, but reduce it in Experiment 2B. Although there is a possibility that such a benefit occurs, it would appear to be a small one at best, given the results of Experiment 1. There, using the same task for pictures and words (Exp. 1A) did not provide any reduction in competitor priming relative to when different tasks were used (Exp. 1B).

\section{General discussion}

Waszak and colleagues (Waszak, 2010; Waszak et al., 2003, 2004, 2005) have clearly demonstrated two separate episodic effects of prior experience on task-switch costs, negative and competitor priming. The theoretical foundation for these priming effects is the proposal that applying an intended action to a stimulus results in an event code that integrates stimulus and action features (Hommel, 1998, 2004, 2009). For competitor priming, the retrieved event code includes the current distractor stimulus in the role of target, the task that had been performed on the distractor, and the relevant response. Our experiments were aimed at the question of whether the task set that was included in the retrieved event code might be misapplied to the actual target, not only the distractor, on the current trial.

The suggested task-based contribution to competitor priming is not likely to be evident if the tasks applied to the target and distractor stimuli are similar in their processing requirements. In such a case, applying the distractor task to the target stimulus might actually assist in the performance of the intended task. Therefore, in Experiment 1A we used picture-naming and wordreading tasks in a task-switching procedure to establish a baseline magnitude for competitor priming. We observed a competitor priming effect of a size (about 40 $\mathrm{ms}$ ) nearly identical to that reported by Waszak et al. (2005, Exp. 1), constituting a close replication of their result. Then, in Experiment 1B, we changed the nature of the task applied to picture stimuli so that subjects were required to make semantic classification responses with button presses. If that task were misapplied to a 
word target on switch trials, when the target was supposed to have been read aloud, then an increased competitor priming effect should be observed relative to what was found in Experiment 1A. Instead, however, we obtained a competitor priming effect similar to, but slightly smaller than, what was found in Experiment $1 \mathrm{~A}$.

It is possible that application of the competing task set to the target stimulus is unlikely to occur unless that task is one that is habitually applied to the target (e.g., reading a word). In the case of word targets, there is no natural tendency for those stimuli to afford a semantic classification decision (the competitor task in Exp. 1B), and this may explain the lack of increased competitor priming in that situation. We tested this possibility in Experiment 2 by changing the task applied to word targets to be the semantic classification task. The task used with pictures was either naming (Exp. 2A) or semantic classification (Exp. 2B). In this case, the latter arrangement served as the baseline for measuring competitor priming when responding to word targets, and we again observed an effect of about $40 \mathrm{~ms}$. But when the naming task was applied to pictures (Exp. 2A), we obtained evidence of misapplication of that task to word targets in the form of enhanced competitor priming (over $90 \mathrm{~ms}$ ). None of the other combinations of tasks and stimulus types that we examined generated such a large competitor priming effect. Moreover, this increase was restricted to competitor priming, as the negative priming effect for word stimuli was virtually the same regardless of the nature of the picture task that was used.

In presenting the comparison of competitor priming effects across our experiments, we have implicitly assumed that the usual mechanism for competitor priming (application of the distractor task to the distractor stimulus) has roughly the same impact in all cases and that the modulation we have reported is the product of an additional mechanism. It is possible, however, that the usual source of competitor priming is somewhat enhanced when the same task is used for both stimulus types; the goal of applying the task to the target stimulus might support the tendency to apply that task to the distractor stimulus. The similar levels of competitor priming for word stimuli obtained in Experiments $1 \mathrm{~A}$ and $1 \mathrm{~B}$ might, then, be a result of a roughly balanced contribution from two different sources of competitor priming that vary in opposite ways in the size of their contributions across the experiments. A stronger contribution would be made by application of the distractor task to the distractor stimulus in Experiment 1A (where distractor and target tasks were the same), but a larger impact of the tendency to apply the distractor task to the target stimulus would have operated in Experiment $1 \mathrm{~B}$ (where the two tasks were different). Although this idea is plausible, we note that in Experiment 2 we did not observe the kind of balanced contributions that on this account might have been at work in Experiment 1 . For now, we prefer the simpler assumption that the influence of applying the distractor task to the distractor stimulus was similar across all four experiments and that applying a distractor task to a target stimulus that naturally affords that task can elevate competitor priming.

We conclude that the event codes underling competitor priming in task switching include elements that may combine with features of the currently relevant task to produce additional competition. It is not clear whether all of the elements of an event code have the potential to recombine with external task components in this way, but we have provided evidence that elements comprising the competing task set are capable of doing so. It appears that in order for the competitor task to combine with the target stimulus, that task must clearly be afforded by the general class of target stimuli, probably through extensive pre-experimental experience. We specify the general class of targets as being involved in this relationship, rather than particular target items, because competitor priming is assessed by comparing two conditions (CP and UP) in which the target stimuli have never appeared as distractors on trials where the competitor task is the correct one to perform. The combination of availability of a competing task set (through retrieval of the competing event code) and the relevance of that task set to the class of target stimuli, then, appears to induce the unintended application of the task to the current target and thereby yields a substantial increase in competitor priming. This form of competitor priming cannot be detected in typical task-switching experiments where a single (bivalent) stimulus is presented on each trial (e.g., a digit in the context of parity and magnitude judgment tasks). Rather, it is necessary to use separate target and distractor stimuli, as was done by Waszak et al. $(2003,2004,2005)$ and in the present experiments. This aspect of competitor priming has the potential to contribute to task-switch costs as well as to overall priming effects in those situations where stimulus compounds are used.

Acknowledgements This research was supported by the Natural Sciences and Engineering Research Council of Canada through a Canada Graduate Scholarship to M. Teskey and a discovery grant to M. Masson. We thank Marnie Jedynak for assistance in conducting the experiments and Jennifer Stolz and two anonymous reviewers for very helpful comments on an earlier version of this article. 


\section{Appendix}

\section{Errors}

The mean percent error in each condition for Experiments 1A, 1B, 2A. and 2B are presented in Table 1. These data were examined using the same Bayesian method as for the response-time data. We first collapsed across prime type and analyzed the influence of trial type (switch, repeat) and target type (word, picture), except in the case of Experiment 1B where the errorless word task was excluded from the analysis. There was no evidence of an effect of trial type on error percent, except in Experiment $2 \mathrm{~A}$ where more errors were made on switch than on repeat trials $(B F=5.7$ in favor of an effect). The error rate was higher for picture targets than for word targets in Experiment $1 \mathrm{~A}(B F=14.6)$, but the reverse was found for Experiments $2 \mathrm{~A}$ and $2 \mathrm{~B}(B F \mathrm{~s}>1000)$. In none of the experiments was there an interaction between trial type and target type ( $B F \mathrm{~s}>1.2$ in favor of the null). The mean effect of each type of priming on error percent is presented in Fig. A1. None of the task arrangements produced reliable priming effects in the negative direction, which might have compromised the interpretation of priming effects in the response-time data by suggesting possible speed-accuracy trade-offs.

\section{Spoils}

Mean percent spoil percent for Experiments 1A, 1B, and 2A are presented in Table 1. Only conditions in which the target task required a verbal response could contain spoils, and other conditions were excluded from analyses. We first collapsed across prime type and analyzed the influence of trial type (switch, repeat) for each experiment. In addition, target type (word, picture) was a factor for Experiment 1A. Spoil rates on repeat trials were higher than spoil rates on switch trials in all cases $(B F \mathrm{~s}>$ 11.7). In Experiment 1A, spoil rates were higher in the picture naming task compared to the word reading task in $(B F>1,000)$, but there was no evidence of an interaction between trial type and target type ( $B F=4.5$, in favor of the null). Priming effects for spoil percent are shown in Fig. A2. No clear priming effects were present, except for a reverse competitor priming effect in Experiment 2A. At this point we do not have an explanation for reduced spoils under competitor priming.
Fig 4 Effect size for percent error relative to the unprimed condition for negative and competitor priming $(\mathrm{NCP})$, competitor priming $(\mathrm{CP})$, and negative priming (NP) as a function of trial type and target type. The word task for Experiment 1B is excluded due to an absence of errors. Error bars represent 95\% highest density intervals

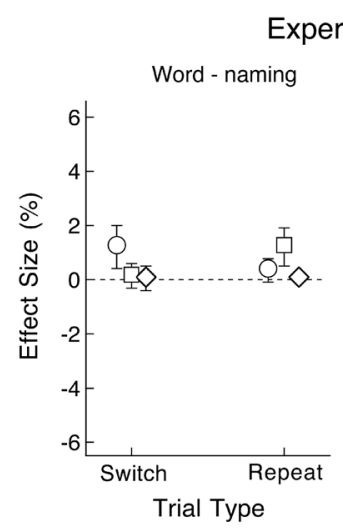

Experiment 1A

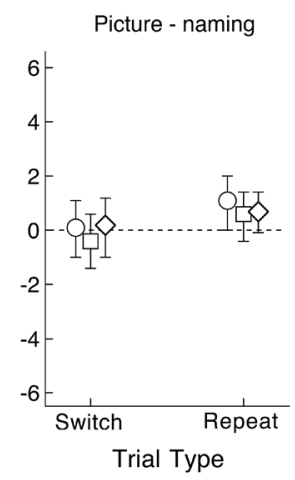

Experiment 2A
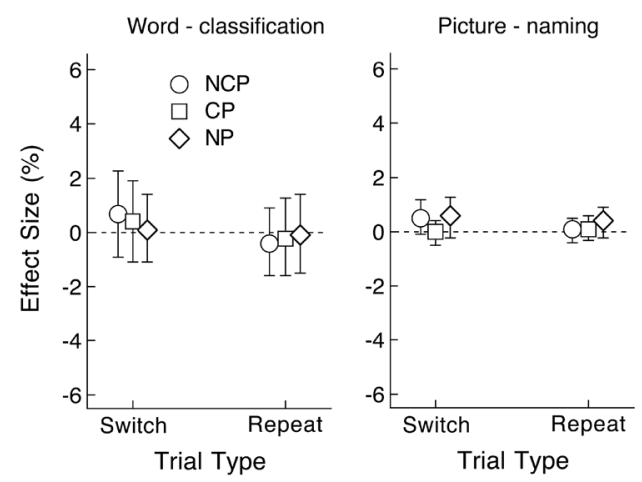
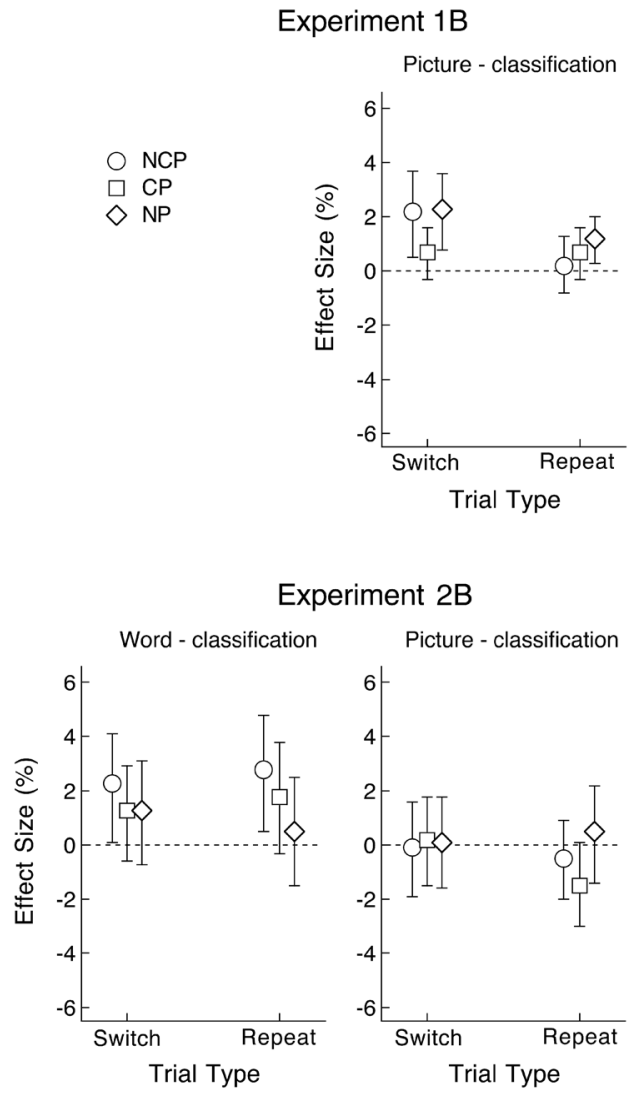

$\bigcirc \mathrm{NCP}$

$\diamond \mathrm{NP}$

Experiment 2B 
Fig 5 Effect size for spoil percent relative to the unprimed condition for negative and competitor priming $(\mathrm{NCP})$, competitor priming $(\mathrm{CP})$, and negative priming (NP) as a function of trial type and target type. This measure is relevant only where the task required vocal responses. Error bars represent $95 \%$ highest density intervals
Exp. $1 \mathrm{~A}$ - Word naming Exp. $1 \mathrm{~A}$ - Picture naming
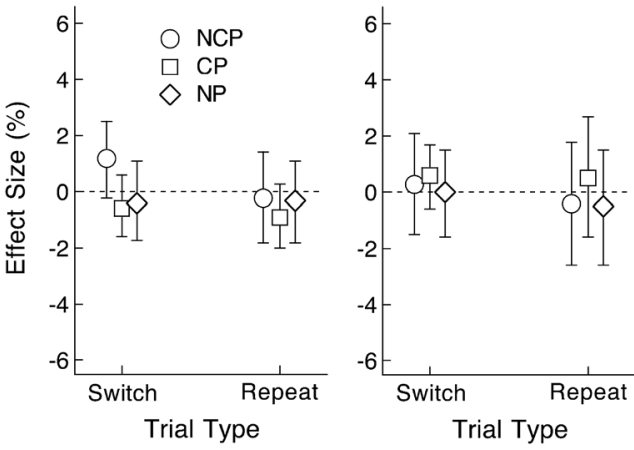

Exp. 1B Word naming

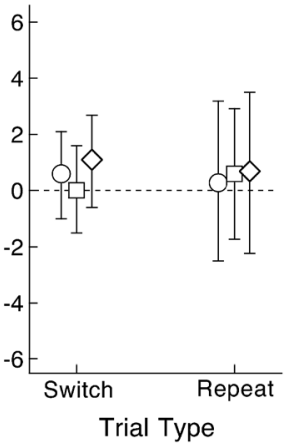

Exp. 2A - Picture naming

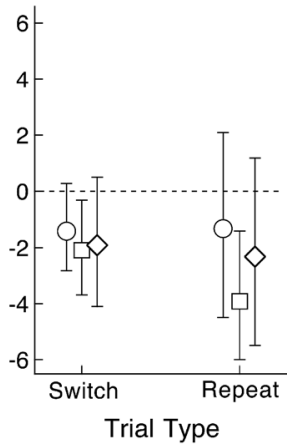

\section{References}

Allport, A. (1987). Selection for action: Some behavioral and neurophysiological considerations of attention and action. In H. Heuer \& A. F. Sanders (Eds.), Perspectives on perception and action (pp. 395419). Hillsdale: Erlbaum.

Allport, A., Styles, E. A., \& Hsieh, S. (1994). Shifting intentional set: Exploring the dynamic control of tasks. In C. Umilta \& M. Moscovitch (Eds.), Attention and performance XV: Conscious and nonconscious information processing (pp. 421-452). Cambridge: MIT Press.

Allport, A., \& Wylie, G. (1999). Task-switching: Positive and negative priming of task-set. In G. W. Humphreys, J. Duncan, \& A. M. Treisman (Eds.), Attention, space, and action: Studies in cognitive neuroscience (pp. 273-296). Oxford: Oxford University Press.

Allport, A., \& Wylie, G. (2000). Task switching, stimulus-response bindings, and negative priming. In S. Monsell \& J. Driver (Eds.), Attention and performance XVIII: Control of cognitive processes (pp. 35-70). Cambridge: MIT Press.

Besner, D., \& Stolz, J. A. (1999). What kind of attention modulates the Stroop effect? Psychonomic Bulletin \& Review, 6, 99-104.

Dreisbach, G., \& Haider, H. (2009). How task representations guide attention: Further evidence for the shielding function of task sets. Journal of Experimental Psychology: Learning, Memory, and Cognition, 35, 477-486.

Dreisbach, G., \& Wenke, D. (2011). The shielding function of task sets and its relaxation during task switching. Journal of Experimental Psychology: Learning, Memory, and Cognition, 37, 1540-1546.

Hommel, B. (1998). Event files: Evidence for automatic integration of stimulus-response episodes. Visual Cognition, 5, 183-216.

Hommel, B. (2004). Event files: Feature binding in and across perception and action. Trends in Cognitive Sciences, 8, 494-500.

Hommel, B. (2009). Action control according to TEC (theory of event coding). Psychological Research, 73, 512-526.

Jersild, A. T. (1927). Mental set and shift. Archives of Psychology, Whole No. 89.

Koch, I., \& Allport, A. (2006). Cue-based preparation and stimulus-based priming of tasks in task switching. Memory \& Cognition, 34, 433444.

Koch, I., Prinz, W., \& Allport, A. (2005). Involuntary retrieval in alphabet-arithmetic tasks: Task-mixing and task-switching costs. Psychological Research, 69, 252-261.
MacLeod, C. M. (1991). Half a century of research on the Stroop effect: An integrative review. Psychological Bulletin, 109, 163-203.

Malley, G. B., \& Strayer, D. L. (1995). Effect of stimulus repetition on positive and negative identity priming. Perception \& Psychophysics, 57, 657-667.

Morey, R., \& Rouder, J. (2015). BayesFactor: Computation of Bayes Factors for Common Designs. R package version 0.9.12-2. http://CRAN.R-project.org/package=BayesFactor

Neill, W. T. (1977). Inhibitory and facilitatory processes in selective attention. Journal of Experimental Psychology: Human Perception and Performance, 3, 444-450.

Raftery, A. E. (1995). Bayesian model selection in social research. In P. V. Marsden (Ed.), Sociological methodology 1995 (pp. 111-196). Cambridge: Blackwell.

Rogers, R. D., \& Monsell, S. (1995). Costs of a predictable switch between simple cognitive tasks. Journal of Experimental Psychology: General, 124, 207-231.

Rouder, J. N., Morey, R. D., Speckman, P. L., \& Province, J. M. (2012). Default Bayes factors for ANOVA designs. Journal of Mathematical Psychology, 56, 356-374.

Rouder, J. N., Morey, R. D., Verhagen, J., Swagman, A. R., \& Wagenmakers, E.-J. (2017). Bayesian analysis of factorial designs. Psychological Methods, 22, 304-321.

Smith, M. C., \& Magee, L. E. (1980). Tracing the time course of pictureword processing. Journal of Experimental Psychology: General, 109, 373-392.

Snodgrass, J. G., \& Vanderwart, M. (1980). A standardized set of 260 pictures: Norms for name agreement, image agreement, familiarity, and visual complexity. Journal of Experimental Psychology: Human Learning and Memory, 6, 174-215.

Stephan, D. N., \& Koch, I. (2010). Central cross-talk in task switching: Evidence from manipulating input-output modality compatibility. Journal of Experimental Psychology: Learning, Memory, and Cognition, 36, 1075-1081.

Stephan, D. N., \& Koch, I. (2011). The role of input-output modality compatibility in task switching. Psychological Research, 75, 491-498.

Stolz, J. A., \& Besner, D. (1996). Role of set in visual word recognition: Activation and activation blocking as nonautomatic processes. Journal of Experimental Psychology: Human Perception and Performance, 22, 1166-1177.

Stroop, J. R. (1935). Studies of interference in serial verbal reactions. Journal of Experimental Psychology, 18, 643-662. 
Tipper, S. P. (1985). The negative priming effect: Inhibitory priming by ignored objects. Quarterly Journal of Experimental Psychology, 37, 571-590.

Ulrich, R., \& Miller, J. (1994). Effects of truncation on reaction time analysis. Journal of Experimental Psychology: General, 123, 34-80.

Waszak, F. (2010). Across-task long-term priming: Interaction of task readiness and automatic retrieval. Quarterly Journal of Experimental Psychology, 63, 1414-1429.

Waszak, F., \& Hommel, B. (2007). The costs and benefits of cross-task priming. Memory \& Cognition, 35, 1175-1186.
Waszak, F., Hommel, B., \& Allport, A. (2003). Task-switching and long-term priming: Role of episodic stimulus-task bindings in task-shift costs. Cognitive psychology, 46, 361-413.

Waszak, F., Hommel, B., \& Allport, A. (2004). Semantic generalization of stimulus-task bindings. Psychonomic Bulletin \& Review, 11, 1027-1033.

Waszak, F., Hommel, B., \& Allport, A. (2005). Interaction of task readiness and automatic retrieval in task switching: Negative priming and competitor priming. Memory \& cognition, 3, 595-610. 